\title{
Sarcophagidae (Insecta, Diptera) associados à decomposição de carcaças de Sus scrofa Linnaeus (Suidae) em área de Cerrado do Distrito Federal, Brasil
}

\author{
Rodrigo Meneses de Barros ${ }^{1}$, Cátia Antunes de Mello-Patiu² \& José Roberto Pujol-Luz ${ }^{1}$
}

\begin{abstract}
${ }^{1}$ Departamento de Zoologia, Instituto de Ciências Biológicas, Universidade de Brasília. 70910-900, Brasília-DF, Brazil. rodrigo_mdebarros@yahoo.com.br; jrpujol@unb.br

${ }^{2}$ Departamento de Entomologia, Museu Nacional, Universidade Federal do Rio de Janeiro. Quinta da Boa Vista, São Cristóvão. 20940-040, Rio de Janeiro-RJ, Brazil. camello@acd.ufrj.br
\end{abstract}

\begin{abstract}
Sarcophagidae (Insecta, Diptera) associated to the decay process of Sus scrofa Linnaeus (Suidae) carcasses in a Cerrado area of Distrito Federal, Brazil. This work presents a list of adult flesh flies collected from a pig carrion Sus scrofa, in a Cerrado area of Distrito Federal, Brazil, being a study on the composition of the dipteran fauna in the region. The field work followed the decay of the carrion and was conduced for 45 days. From 4,626 collected flesh flies, 28 species were identified, consisting in 16 new records for this region.
\end{abstract}

KEYWORDS. Checklist; flesh flies; forensic entomology.

RESUMO. Sarcophagidae (Insecta, Diptera) associados à decomposição de carcaças de Sus scrofa Linnaeus (Suidae) em área de Cerrado do Distrito Federal, Brasil. Este trabalho apresenta uma lista de dípteros sarcofagídeos adultos coletados em carcaça de porco Sus scrofa, em área de Cerrado, Distrito Federal, Brasil, sendo um estudo sobre a composição da fauna de Diptera na região. O trabalho de campo acompanhou o processo de decomposição da carcaça e foi realizado durante 45 dias. A partir de 4.626 indivíduos coletados foram identificadas 28 espécies, sendo 16 novos registros para a região estudada.

PALAVRAS-CHAVE. Entomologia forense; lista; sarcofagídeos.

Os Sarcophagidae possuem cerca de 2.600 espécies descritas (Pape 1996) e juntamente com Calliphoridae, Muscidae, Fanniidae e Stratiomyidae, são de grande importância para a Entomologia Forense (Payne 1965; Smith 1986; Early \& Goff 1986; Tullis \& Goff 1987; Catts \& Goff 1992). A fauna neotropical é bastante diversa, com mais de 750 espécies descritas (Pape 1996), com vários trabalhos que relatam a ocorrência de sarcofagídeos associados a carcaças de animais vertebrados no Brasil (Lopes 1973; Monteiro-Filho \& Penereiro 1987; Salviano 1996; Moura et al. 1997; Carvalho et al. 2000; Carvalho \& Linhares 2001), incluindo cadáveres humanos (Freire 1923; Carvalho et al. 2000; Oliveira-Costa et al.2001).

A maioria das espécies é ovovivípara, eliminando larvas de primeiro instar, que iniciam imediatamente sua alimentação na carcaça (Denno \& Cothran 1976). Essa estratégia de desenvolvimento se distingue daquela dos califorídeos, que são ovíparos e necessitam de um tempo adicional para a eclosão dos ovos, além de ovipositarem em locais específicos na carcaça. Acreditamos que a diferença nas estratégias das duas famílias pode conferir aos Sarcophagidae um pioneirismo na colonização dos cadáveres, o que ressalta a sua importância forense.

Neste trabalho são apresentados pela primeira vez os resultados de uma investigação sobre a fauna de Sarcophagidae atraída por uma carcaça de porco exposta em área de Cerrado, no Distrito Federal, Brasil.

\section{MATERIALEMÉTODOS}

O experimento foi desenvolvido na Fazenda Água Limpa, da Universidade de Brasília, no Distrito Federal. O sítio experimental possui uma área de cerca de $1.600 \mathrm{~m}^{2}\left(15^{\circ} 56^{\prime} 22^{\prime \prime} \mathrm{S}\right.$ / 47 54'54" O) e consiste em uma pastagem desativada, rodeada por Cerrado sensu stricto.

Para atratividade dos insetos foi utilizada uma carcaça de porco macho em decomposição (Sus scrofa Linnaeus, 1758), com aproximadamente $15 \mathrm{~kg}$, sacrificado com um tiro de calibre 22 na região da cabeça. Durante a etapa experimental, a carcaça foi mantida em condições naturais no interior de uma gaiola de metal, para evitar o acesso de animais carniceiros, e coberta por uma armadilha entomológica do tipo Shannon modificada, com 1,5 m de diâmetro por dois metros de altura, suspensa cerca de $10 \mathrm{~cm}$ do solo, o que facilitou a entrada das moscas em seu interior. Foram consideradas cinco fases de decomposição conforme Early \& Goff (1986): fresca, inchamento, deterioração, seca e restos.

As coletas duraram cerca de 15 minutos e foram realizadas diariamente nos períodos de $1^{\circ}$ a 30 de junho e semanalmente, de $1^{\circ}$ a 15 de julho de 2004 , quando a carcaça atingiu o estágio 
de restos e a presença de adultos foi muito reduzida. Os insetos foram capturados no interior da armadilha com auxílio de redes entomológicas e sacos plásticos.

Pela carência de chaves de identificação para os gêneros neotropicais e pelo conhecimento ainda incipiente da fauna do Cerrado, a identificação das espécies coletadas foi realizada por comparação com material da coleção do Museu Nacional/ UFRJ e com base em Lopes (1945; 1958; 1976a; 1976b); Tibana \& Mello (1983); Lopes \& Tibana (1987), Leite \& Lopes (1989), Lopes \& Leite (1991); Tibana \& Mello (1992) e Mello (1996). Devido à dificuldade taxonômica apresentada pelo grupo, a identificação foi baseada nos machos. A classificação das espécies de Sarcophagidae seguiu a proposta de Pape (1996). O material foi depositado na Coleção Entomológica do Departamento de Zoologia da Universidade de Brasília.

\section{RESULTADOS}

Foram coletados 4.626 Sarcophagidae, com uma relação entre os sexos de aproximadamente um macho para cada três fêmeas. A tabela I apresenta a abundância das 28 espécies identificadas, que totalizam 1344 espécimes machos.

As espécies de Oxysarcodexia Townsend somaram 42,48\% do total. Dentre elas, O. thornax apresentou a maior abundância, seguida por Dexosarcophaga carvalhoi, Tricharea (Sarcophagula) occidua e Sarcodexia lambens. Dentre as espécies de Peckia Robineau-Desvoidy, destacouse Peckia (Peckia) pexata como a mais abundante.

As cinco fases de decomposição propostas por Early \& Goff (1986) foram observadas, sendo que as fases fresca, inchamento, deterioração e seca duraram um, oito, 13 e nove dias, respectivamente. As coletas foram finalizadas 14 dias após a entrada na fase de restos. A maioria dos Sarcophagidae foi coletada durante a fase de inchamento (Tabela II).

Considerando ambos os sexos, foram coletados 2.947 sarcofagídeos $(63,70)$ no intervalo entre o $7^{\circ}$ e o $14^{\circ}$ dia após a morte do animal, período compreendido nas fases de inchamento e deterioração da carcaça. O pico populacional ocorreu no $10^{\circ}$ dia após a morte do animal com 509 sarcofagídeos coletados, $11,00 \%$ do total.

\section{DISCUSSÃO}

A alta diversidade de Sarcophagidae em carcaças no Brasil (Salviano 1996; Carvalho \& Linhares 2001; Oliveira-Costa et al. 2001; Oliveira-Costa 2003) foi constatada neste trabalho. Salviano (1996) estudou a dipterofauna atraída por carcaças de porcos em uma área urbana, arborizada, na cidade do Rio

Tabela I. Abundância dos adultos machos de Sarcophagidae coletados em carcaça de porco em área de Cerrado, na cidade de Brasília, no período de 01 de junho a 15 de julho de 2004 .

\begin{tabular}{|c|c|c|c|}
\hline Espécies & Abundância absoluta & Abundância relativa & Novo registro \\
\hline Argoravinia rufiventris (Walker, 1849) & 5 & $0,37 \%$ & $\mathrm{x}$ \\
\hline Dexosarcophaga ampullula (Engel, 1931) & 4 & $0,30 \%$ & \\
\hline Dexosarcophaga carvalhoi (Lopes, 1980) & 396 & $29,46 \%$ & $\mathrm{x}$ \\
\hline Dexosarcophaga lenkoi Lopes, 1968 & 8 & $0,60 \%$ & $\mathrm{x}$ \\
\hline Dexosarcophaga paulistana (Lopes, 1982) & 1 & $0,07 \%$ & $\mathrm{x}$ \\
\hline Dexosarcophaga sp. 1 & 4 & $0,30 \%$ & \\
\hline Dexosarcophaga sp. 2 & 1 & $0,07 \%$ & \\
\hline Helicobia aurescens (Townsend, 1927) & 1 & $0,07 \%$ & $\mathrm{x}$ \\
\hline Oxysarcodexia admixta (Lopes, 1933) & 1 & $0,07 \%$ & \\
\hline Oxysarcodexia aura (Hall, 1937) & 3 & $0,22 \%$ & \\
\hline Oxysarcodexia avuncula (Lopes, 1933) & 32 & $2,38 \%$ & \\
\hline Oxysarcodexia diana (Lopes, 1933) & 18 & $1,34 \%$ & $\mathrm{x}$ \\
\hline Oxysarcodexia eberti Lopes \& Tibana, 1987 & 14 & $1,04 \%$ & $\mathrm{x}$ \\
\hline Oxysarcodexia fluminensis Lopes, 1946 & 1 & $0,07 \%$ & $\mathrm{x}$ \\
\hline Oxysarcodexia paulistanensis (Mattos, 1919) & 2 & $0,15 \%$ & $\mathrm{x}$ \\
\hline Oxysarcodexia thornax (Walker, 1849) & 500 & $37,20 \%$ & \\
\hline Peckia (Euboettcheria) anguilla (Curran \& Walley, 1934) & 17 & $1,26 \%$ & $\mathrm{x}$ \\
\hline Peckia (Euboettcheria) collusor (Curran \& Walley, 1934) & 24 & $1,79 \%$ & $\mathrm{x}$ \\
\hline Peckia (Pattonella) intermutans (Walker, 1861) & 5 & $0,37 \%$ & \\
\hline Peckia (Peckia) chrysostoma (Wiedemann, 1830) & 3 & $0,22 \%$ & $\mathrm{x}$ \\
\hline Peckia (Peckia) pexata (Wulp, 1895) & 59 & $4,39 \%$ & $\mathrm{x}$ \\
\hline Peckia (Squamatodes) ingens (Walker, 1849) & 1 & $0,07 \%$ & $\mathrm{x}$ \\
\hline Peckia (Squamatodes) trivittata (Curran, 1927) & 14 & $1,04 \%$ & \\
\hline Ravinia belforti (Prado \& Fonseca, 1932) & 6 & $0,45 \%$ & \\
\hline Sarcodexia lambens (Wiedemann, 1830) & 95 & $7,07 \%$ & $\mathrm{x}$ \\
\hline Sarcophaga (Lipoptilocnema) sp.nov. & 1 & $0,07 \%$ & \\
\hline Titanogrypa (Cucullomyia) larvicida (Lopes, 1935) & 1 & $0,07 \%$ & $\mathrm{x}$ \\
\hline Tricharea (Sarcophagula) occidua (Frabricius, 1794) & 127 & $9,45 \%$ & \\
\hline Total & 1.344 & $100,00 \%$ & 16 \\
\hline
\end{tabular}


Tabela II. Número de adultos machos de Sarcophagidae atraídos por cada fase de decomposição da carcaça de porco em área de Cerrado, na cidade de Brasília, no período de 01 de junho a 15 de julho de 2004.

\begin{tabular}{|c|c|c|c|c|c|}
\hline Espécies & Fresco & Inchamento & Deterioração & Seco & Restos \\
\hline A. rufiventris & 0 & 4 & 1 & 0 & 0 \\
\hline D. ampullula & 0 & 3 & 0 & 0 & 1 \\
\hline D. carvalhoi & 8 & 210 & 154 & 14 & 10 \\
\hline D. lenkoi & 0 & 4 & 3 & 1 & 0 \\
\hline D. paulistana & 0 & 1 & 0 & 0 & 0 \\
\hline D. sp. 1 & 0 & 3 & 1 & 0 & 0 \\
\hline D. sp. 2 & 0 & 1 & 0 & 0 & 0 \\
\hline H. aurescens & 0 & 0 & 1 & 0 & 0 \\
\hline O. admixta & 0 & 1 & 0 & 0 & 0 \\
\hline O. aura & 0 & 3 & 0 & 0 & 0 \\
\hline O. avuncula & 0 & 16 & 9 & 4 & 3 \\
\hline O. diana & 1 & 8 & 8 & 1 & 0 \\
\hline O. eberti & 0 & 3 & 7 & 4 & 0 \\
\hline O. fluminensis & 0 & 1 & 0 & 0 & 0 \\
\hline O. paulistanensis & 0 & 2 & 0 & 0 & 0 \\
\hline O. thornax & 11 & 250 & 163 & 55 & 21 \\
\hline P. (E.) anguilla & 0 & 8 & 8 & 1 & 0 \\
\hline P. (E.) collusor & 0 & 8 & 13 & 2 & 1 \\
\hline P. (P.) intermutans & 0 & 3 & 2 & 0 & 0 \\
\hline P. (P.) chrysostoma & 0 & 1 & 2 & 0 & 0 \\
\hline P. (P.) pexata & 0 & 33 & 24 & 2 & 0 \\
\hline P. (S.) ingens & 0 & 1 & 0 & 0 & 0 \\
\hline P. (S.) trivittata & 0 & 13 & 1 & 0 & 0 \\
\hline R. belforti & 0 & 1 & 3 & 2 & 0 \\
\hline S. lambens & 0 & 16 & 58 & 19 & 2 \\
\hline S. (L.) sp. nov. & 0 & 1 & 0 & 0 & 0 \\
\hline T. (C.) larvicida & 0 & 0 & 1 & 0 & 0 \\
\hline T. (S.) occidua & 0 & 59 & 45 & 22 & 1 \\
\hline Total & 20 & 654 & 504 & 127 & 39 \\
\hline
\end{tabular}

de Janeiro e registrou 19 espécies de Sarcophagidae. Carvalho \& Linhares (2001) registraram 21 espécies dessa família em estudo semelhante realizado em uma reserva florestal na cidade de Campinas, São Paulo. As seguintes espécies registradas pelos autores nos estados do Rio de Janeiro e São Paulo, respectivamente, foram encontradas neste trabalho: Oxysarcodexia diana, O. thornax, Peckia (Euboettcheria) anguilla, P. (E.) collusor, P. (Pattonella) intermutans, Ravinia belforti e $S$. lambens.

Oxysarcodexia e Peckia são gêneros que possuem alta riqueza e abundância em carcaças no Brasil (Salviano 1996; Carvalho \& Linhares 2001). Dentre suas espécies, O. avuncula, $O$. fluminensis, $O$. thornax, P. intermutans e $P$. chrysostoma já foram coletadas em cadáveres humanos no Brasil.

O gênero Oxysarcodexia apresentou o maior número de espécies (oito) e maior abundância, resultado semelhante ao de Salviano (1996), que obteve Oxysarcodexia como o gênero mais abundante na cidade de Rio de Janeiro. O. thornax foi a espécie mais abundante e, juntamente com $D$. carvalhoi, ocorreu em todas as fases de decomposição da carcaça.

Peckia foi o segundo gênero mais diversificado neste estudo, com sete espécies, resultado semelhante ao obtido por Carvalho \& Linhares (2001), que citaram oito espécies deste gênero encontradas em carcaças de porco na cidade de Campinas, São Paulo. Esse gênero possui espécies cujas larvas são freqüentemente encontradas em cadáveres humanos, recebendo grande atenção, principalmente para estudos sobre aspectos bionômicos (Salviano et al. 1996; Ferraz 1998; Oliveira et al. 2002; Loureiro et al. 2005; Oliveira-da-Silva et al. 2006).

Dexosarcophaga Townsend é pela primeira vez associado a carcaças de porcos no Brasil, com cinco espécies encontradas. Pouco se conhece sobre os hábitos das espécies deste gênero. Acredita-se que necessitem de um ambiente especial para o desenvolvimento das larvas, pois têm sido encontradas relacionadas a ninhos de formigas e cupins, além de galerias das larvas (brocas) de curculionídeos (Lopes 1968). D. carvalhoi parece ser uma espécie típica de Cerrado, sua localidade-tipo é Pirapora, Minas Gerais, e tem sido identificada comumente em material proveniente desta região.

Diferentemente do observado por Carvalho \& Linhares (2001), o presente estudo não demonstra uma associação dos Sarcophagidae com estágios avançados de decomposição, mas sim uma maior abundância e diversidade na fase de inchamento da carcaça.

Embora não tenham sido coletadas larvas durante este estudo, em experimento semelhante realizado no mesmo local (Barros et al. 2006), foi observado que larvas de P. trivittata foram pioneiras na colonização, conseguindo se alimentar e abandonar a carcaça para empupar antes que as larvas de Calliphoridae atingissem o terceiro instar. Fato semelhante foi observado por Salviano (1996) para P. intermutans.

Ressaltamos que o conhecimento das espécies envolvidas no processo de decomposição é o primeiro passo para uma efetiva utilização dos insetos como ferramenta para resolução de crimes. A lista de espécies de Sarcophagidae apresentada neste trabalho revela a grande diversidade deste grupo de insetos e reforça sua importância em estudos sobre a fauna cadavérica que ocorre em áreas de Cerrado, sobretudo no Distrito Federal.

Agradecimentos. Ao Prof. Fernando Henrique de Assis Santana pelo incentivo, auxílio nas coletas e triagem do material. Aos colegas e funcionários da Fazenda Água Limpa da Universidade de Brasília, pelo suporte técnico. Ao programa institucional de Bolsas de Iniciação Científica da Universidade de Brasília pela concessão da bolsa PIBIC/ $\mathrm{CNPq}$ (RMB). Ao Conselho Nacional de Pesquisa e Desenvolvimento Tecnológico, $\mathrm{CNPq}$ pela bolsa de produtividade em pesquisa e auxílio integrado, processos 308636/07-4 e 474081/07-9 (JRPL). À Fundação Carlos Chagas Filho de Amparo à Pesquisa do Estado do Rio de Janeiro - FAPERJ, pelo auxílio concedido (CAMP). À Comissão de Ética do Instituto de Ciências Biológicas pela autorização concedida para realização do experimento.

\section{REFERÊNCIAS}

Barros, R. M. de; A. M. Penteado-Dias \& J. R. Pujol-Luz. 2006. Registro de Peckia (Squamatodes) trivittata (Curran, 1927) (Diptera, Sarcophagidae) parasitada por Gnathopleura semirufa (Brullé, 1846) (Hymenoptera, Braconidae, Alysiinae) no cerrado de Brasília, DF. Revista Brasileira de Entomologia 50: 436-438.

Carvalho, L. M. L. \& A. X. Linhares. 2001. Seasonality of insect succession and pig carcass decomposition in a natural forest area in 
southeastern Brazil. Journal of Forensic Sciences 46: 604608.

Carvalho, L. M. L.; P. J. Thyssen; A. X. Linhares \& F. A. B. Palhares. 2000. A checklist of arthropods associated with pig carrion and human corpses in Southeastern Brazil. Memórias da Fundação Oswaldo Cruz 95: 135-138.

Catts, E. P. \& M. L. Goff. 1992. Forensic Entomology in criminal investigation. Annual Review of Entomology 37: 253-272.

Denno, R. F. \& W. R. Cothran. 1976. Competitive interactions and ecological strategies of Sarcophagid and Calliphorid flies inhabiting rabit carrion. Annals of the Entomological Society of America 69: 109-113.

Early, M. \& M. L. Goff. 1986. Arthropod succession patterns in exposed carrion on the island of O'ahu, Hawaiian Islands, USA. Journal of Medical Entomology 23: 520-531.

Ferraz, M. V. 1998. Larviposition and rearing media preferences of Peckia chrysostoma and Adiscochaeta ingens (Diptera, Sarcophagidae) larvae reared under laboratory conditions. Revista Brasileira de Entomologia 41: 225-228.

Freire, O. 1923. Fauna cadavérica brasileira. Revista de Medicina 3: $15-40$.

Leite, A. C. R. \& H. S. Lopes. 1989. Studies on male genitalia of Sarcophagidae (Diptera) based on scanning electron microscope observations. Memórias do Instituto Oswaldo Cruz 84: 189199.

Lopes, H. S. 1945. Contribuição ao conhecimento das espécies do gênero Oxysarcodexia Townsend, 1917. Boletim da Escola Nacional de Veterinária 1: 62-136.

Lopes, H. S. 1958. Considerações sobre as espécies de Peckia Desvoidy, 1930 e de gêneros afins (Diptera-Sarcophagidae). Anais da Academia Brasileira de Ciências 30: 212-243.

Lopes, H. S. 1968. Sobre uma espécie nova de "Dexosarcophaga" Townsend, 1917 (Dipt., Sarcophagidae) cujas larvas vivem em ninho de "Camponotus" (Hymenoptera, Formicidae). Revista Brasileira de Biologia 28: 521-523.

Lopes, H. S. 1973. Collecting and rearing sarcophagid flies (Diptera) in Brazil during forty years. Anais da Academia Brasileira de Ciências 45: 279-299.

Lopes, H. S. 1976a. On the species of Argoravinia Townsend (Diptera, Sarcophagidae). Revista Brasileira de Biologia 36: 693-696.

Lopes, H. S. 1976b. On the genus Cuculomyia Roback (Diptera, Sarcophagidae). Revista Brasileira de Biologia 36: 745-757.

Lopes, H. S. \& R. Tibana. 1987. On Oxysarcodexia (Diptera, Sarcophagidae), with descriptions of five new species, key, list and geographic distribution of the species. Revista Brasileira de Biologia 47: 329-347.

Lopes. H. S. \& A. C. R. Leite. 1991. Notes on the male genitalia of species of Ravinia and Chaetoravinia (Diptera: Sarcophagidae). Memórias do Instituto Oswaldo Cruz 86: 95-101.

Loureiro, M. S.; V. C. Oliveira \& J. M. d'Almeida. 2005. Desenvolvimento pós-embrionário de Pattonella intermutans
(Thomson) (Diptera: Sarcophagidae) em diferentes dietas. Revista Brasileira de Entomologia 49: 127-129.

Mello, C. A. 1996. Revisão do gênero Farrimyia Dodge, 1965 (Diptera, Sarcophagidae) - Parte II. Revista Brasileira de Biologia 56: 473-483.

Monteiro-Filho, E. K. L. A. \& J. L Penereiro. 1987. Estudo da decomposição e sucessão sobre uma carcaça animal numa área do estado de São Paulo, Brasil. Revista Brasileira de Biologia 47: $289-285$.

Moura, M. O.; C. J. B. Carvalho \& E. L. A. Monteiro-Filho. 1997. A preliminary analysis of insects of medico-legal importance in Curitiba, State of Paraná. Memórias do Instituto Oswaldo Cruz 92: $269-274$.

Oliveira, V. C.; R. P. Mello \& R. F. S. Santos. 2002. Bionomics aspects of Pattonella intermutans (Thomson, 1869) (Diptera, Sarcophagidae) under laboratory conditions. Brazilian Archives of Biology and Technology 45: 473-477.

Oliveira-Costa, J. 2003. Entomologia Forense - Quanto os insetos são Vestígios. Millenium, $257 \mathrm{p}$.

Oliveira-Costa, J.; C. A. Mello-Patiu \& S. M. Lopes. 2001. Dípteros muscóides associados com cadáveres humanos no local da morte no estado do Rio de Janeiro, Brasil. Boletim do Museu Nacional 464: $1-6$.

Oliveira-da-Silva, A.; R. Ale-Rocha \& J. A. Rafael. 2006. Bionomia dos estágios imaturos de duas espécies de Peckia (Diptera, Sarcophagidae) em suíno em decomposição em área de floresta no norte do Brasil. Revista Brasileira de Entomologia 50: 524-527.

Pape, T. 1996. Catalogue of the Sarcophagidae of the world (Insecta: Diptera). Memoirs of Entomology, International 8: 558 p.

Payne, J. A. 1965. A summer carrion study of the baby pig Sus scrofa Linnaeus. Ecology 46: 592-602.

Salviano, R. J. B. 1996. Sucessão de Diptera Caliptrata em carcaça de Sus scrofa L. Dissertação de Mestrado. Universidade Federal Rural do Rio de Janeiro. 124 p.

Salviano, R. J. B.; R. P. Mello; L. C. N. H. Beck \& J. M. d'Almeida. 1996. Aspectos bionômicos de Squamatoides trivittatus (Diptera, Sarcophagidae) sob condições de laboratório. Memórias do Instituto Oswaldo Cruz 91: 249-254.

Smith, K. G. V. 1986. A manual of Forensic Entomology. British Museum (Natural History) London. 205 p.

Tibana, R. \& C. A. Mello. 1983. Redescrição de Oxysarcodexia thornax (Walker, 1849) e descrição de O. morretesi, sp. n. (Diptera, Sarcophagidae). Revista Brasileira de Entomologia 27: 277283.

Tibana, R. \& C. A. Mello. 1992. Sobre Helicobia aurescens Townsend, 1927 (Diptera, Sarcophagidae). Revista Brasileira de Biologia 52: $45-51$.

Tullis, K. \& M. L. Goff. 1987. Arthropod succession in exposed carrion in a tropical rainforest on O'ahu Island, Hawwaii's. Journal of Medical Entomology 24: 332-339. 